\title{
Adjusted Viterbi Training
}

\author{
Jüri Lember \\ Tartu University, Liivi 2-507, Tartu 50409, Estonia; jyril@ut.ee \\ Alexey Koloydenko* \\ Eurandom, P.O. Box 513 - 5600 MB Eindhoven, The Netherlands. \\ Tel: +31(0)40.247.8129, koloidenko@eurandom.tue.nl \\ http://euridice.tue.nl/ akoloide/VA/
}

January 20, 2004

\begin{abstract}
We propose modifications of the Viterbi Training (VT) algorithm to estimate emission parameters in Hidden Markov Models (HMM) which are widely used in speech recognition, natural language modeling, image analysis, and bioinformatics. Our goal is to alleviate the inconsistency of VT while controlling the amount of extra computations. Specifically, we modify VT to enable it asymptotically to fix the true values of the parameters as does the EM algorithm. Our approach relies on infinite Viterbi alignment and an associated with it limiting probability distribution. We focus on mixture models, an important special case of HMM, wherein the limiting distribution can be computed exactly and be used in the adjusted VT algorithm.

A simulation experiment shows that our central algorithm (VA1) can dramatically improve accuracy without much cost in computation time.

We also propose VA2, a more mathematically advanced correction to VT, verify its fast convergence and high accuracy, and intend to elaborate on its computationally feasible implementations in future work.
\end{abstract}

\section{Introduction}

Motivated by applications of HMMs in speech processing and recognition $\sqrt{6}$, , 1, 14, 18, 19, 20, 22, 23, natural language modeling [15], image analysis [11, 12], bioinformatics [1, 16], and by connections with constrained vector quantization [3, 5], we study estimation of the emission parameters $\theta_{l}$ in hidden Markov models with states $l \in S=\{1, \ldots, K\}$.

The standard method to compute a maximum likelihood estimator of the emission parameters $\theta$, is the EM algorithm that in the HMM context is also known as the Baum-Welch or forward-backward algorithm [1, 2, 6, 7, 18, 19. Since EM can in practice be computationally expensive, it is commonly replaced by Viterbi Training. VT effectively replaces the computationally costly expectation (E) step of EM by an appropriate maximization step that is computationally less intensive. For example, VT is successfully used in Philips speech recognition systems [14.

The price of the computational gain is loss of accuracy: - the VT estimators need not be (local) maximum likelihood estimators (VT does not necessarily increase the likelihood); they are also generally biased and inconsistent.

Given current parameters, VT first finds a Viterbi alignment, a sequence of hidden states that maximizes the likelihood of the observed data. Observations assumed to have been emitted from state $l$, are regarded as an i.i.d. sample from $P_{l}$, the corresponding emission distribution. These observations

${ }^{*}$ Corresponding author 
produce $\hat{P}_{l}^{n}$, the empirical version of $P_{l}$, and ultimately $\hat{\mu}_{l}$, a maximum likelihood estimate of $\theta_{l}$. $\hat{\mu}$ is then used to find an alignment in the next step, and so forth. It can be shown that in general this procedure converges in finitely many steps; also, it is usually much faster than EM.

In speech recognition, the same training procedure was already described by L. Rabiner et al. in [8, 20] (see also [18, 19]) who considered his procedure as a variation of the Lloyd algorithm from vector quantization, and referred to it as segmential K-means training. The analogy with vector quantization is especially pronounced when the underlying chain is a sequence of i.i.d. variables in which case the observations are simply an i.i.d. sample from a mixture distribution ( $\left(33_{1}\right)$. For such mixture models, Viterbi training was also described by R. Gray et al. in [3], where the training algorithm was considered in the vector quantization context under the name of entropy constrained vector quantization (ECVQ). (See also [5] for more recent developments in this theory.)

Our goal is to alleviate the inconsistency of the VT estimators while preserving the good properties (fast convergence and computational feasibility) of the baseline VT algorithm. To this end, we note that $\theta^{*}$, the true parameters, are asymptotically a fixed point of EM but not of VT $\$ 2$. $\$ 3$. We thus attempt to adjust VT in order to restore this property by studying the asymptotics of $\hat{P}_{l}^{n}$. Thus, we discuss the existence of $Q_{l} l \in S$

$$
\hat{P}_{l}^{n} \Rightarrow Q_{l}, \quad l \in S \quad \text { a.s. },
$$

first in the general HMM context $-\S 2$, and then in the special case of mixture models - $\S$. If such limiting measures exist, then under certain continuity assumptions, the estimators $\hat{\mu}_{l}$ will converge to $\mu_{l}$, where

$$
\mu_{l}=\arg \max _{\theta_{l}} \int \ln f_{l}\left(\theta_{l}, x\right) Q_{l}(d x) .
$$

Taking into account the difference between $\mu_{l}$ and the true parameter, the appropriate adjustment of the Viterbi training can now be defined $(\$ 2)$.

However, the asymptotic behavior of $\hat{P}_{l}^{n}$ is not in general straightforward and its analysis requires an extension of the definition of Viterbi alignment at infinitum [10]. With the infinite alignment one can prove the existence of the limiting measures $Q_{l}$ (11), which is essential for the general definition of the adjusted Viterbi training.

To implement these ideas in practice, a closed form of $Q_{l}$ (or $\hat{\mu}_{l}$ ) as a function of the true parameters is necessary. The measures $Q_{l}$ also depend on the transition as well as on the emission models, and computing $Q_{l}$ can be very difficult. However, in the special case of mixture models $\$$, the measures $Q_{l}$ are easier to find. In \$3, we describe the adjusted Viterbi training (VA1) for the mixture case, which we view as the main contribution of this paper: VA1 recovers the asymptotic fixed point property and, since its adjustment function does not depend on data, each iteration of VA1 enjoys the same order of computational complexity (in terms of the sample size) as VT. Moreover, for commonly used mixtures, such as, for example (Example 3.1), mixtures of multivariate normal distributions with unknown means and known covariances, the adjustment function is available in a closed form requiring integration with the mixture densities. Depending on the dimension of the emission variates and on the number of components, and on the available computational resources, one can vary the accuracy of the adjustment. We reiterate that, unlike the computations of the EM algorithm, computations of the adjustment do not involve evaluation and subsequent summation of the mixture density at every data point.

We first introduce these ideas for the case of known mixture weights and then extend them in $\S 4.1$ to the case of unknown weights.

To support our theory, in $\$$ 列 we simulate the mixture of two univariate normal distributions with unit variance, unknown means, and unequal but comparable weights. The main goal of our simulations is to compare the performances of VT, VA1, and EM in terms of the accuracy, convergence, amount of computations per iteration, and the total amount of computations. The simulations are performed under different conditions on the initial guess when the weights are assumed to be known, $\S 5$.1, and unknown, $\S 5.2$, and the results $(\S 5.3, \S[6)$ are consistently in favor of VA1.

In $\S$, we propose VA2, a more mathematically advanced correction to VT; we verify its fast convergence and high accuracy on the simulated data in $\oint$ 同, and intend to elaborate on its computationally 
feasible implementations in future work.

A concluding summary is given in 8 .

\section{General HMM}

We consider the general case of a hidden Markov model and define appropriate adjustments of the baseline Viterbi Training algorithm.

Thus, $Y$ is a Markov chain with finite state space $S$. We assume $Y$ to be irreducible and aperiodic with transition matrix $P=\left(p_{i j}\right)$ and initial distribution $\pi$ that is also the stationary distribution of $Y$. To every state $l \in S$ there corresponds an emission distribution $P_{l}$ on $(\mathcal{X}, \mathcal{B})$, a separable metric space and the corresponding Borel $\sigma$-algebra. Let $f_{l}$, the density of $P_{l}$ with respect to some reference measure $\lambda$ (for instance, the Lebesgue measure), be known up to the parametrization $f_{l}\left(x ; \theta_{l}\right)$. When $Y$ is in state $l$, an observation according to $P_{l}\left(\theta^{*}\right)$ and independent of everything else is emitted, with $\theta^{*}=\left(\theta_{1}^{*}, \ldots, \theta_{K}^{*}\right)$ being the unknown true parameters.

Thus, for any $y=y_{1}, y_{2}, \ldots$, a realization of $Y$, there corresponds a sequence of independent random variables, $X_{1}, X_{2}, \ldots$, where $X_{n}$ has distribution $P_{y_{n}}$. Note that we only observe $X=X_{1}, X_{2}, \ldots$ and the realization $y$ is unknown ( $Y$ is hidden).

The distribution of $X$ is completely determined by the chain parameters $(P, \pi)$ and the emission distributions $P_{l}, l \in S$.

The process $X$ is also mixing and, therefore, ergodic.

We now recall Viterbi Alignment and Training.

Let $x_{1}, \ldots, x_{n}$ be first $n$ observations on $X$. Let $\Lambda\left(q_{1}, \ldots, q_{n} ; x_{1}, \ldots, x_{n} ; \theta\right)$ be the likelihood function $\mathbf{P}\left(Y_{i}=q_{i}, i=1, \ldots, n\right) \prod_{i=1}^{n} f_{q_{i}}\left(x_{i} ; \theta_{q_{i}}\right), q_{i} \in S$.

The Viterbi alignment is any sequence of states $q_{1}, \ldots, q_{n} \in S$ that maximizes the likelihood of observing $x_{1}, \ldots, x_{n}, \theta$ being fixed. Thus, for a fixed $\theta$, the Viterbi alignment is a maximum-likelihood estimator of the realization of $Y_{1}, \ldots, Y_{n}$ given $x_{1}, \ldots, x_{n}$. In the following, the Viterbi alignment will be referred to as the alignment. For each $n \geq 1$, let $\mathcal{V}$ be the set of alignments:

$$
\mathcal{V}\left(x_{1}, \ldots, x_{n} ; \theta\right)=\left\{v \in S^{n}: \forall w \in S^{n} \Lambda\left(v ; x_{1}, \ldots, x_{n} ; \theta\right) \geq \Lambda\left(w ; x_{1}, \ldots, x_{n} ; \theta\right)\right\} .
$$

Any map $v: \mathcal{X}^{n} \mapsto \mathcal{V}\left(x_{1}, \ldots, x_{n} ; \theta\right)$ will also be called an alignment. Further, unless explicitly specified, $v_{\theta}$ will denote an arbitrary element of $\mathcal{V}\left(x_{1}, \ldots, x_{n} ; \theta\right)$.

\section{$\underline{\text { Viterbi Training }}$}

1.) Choose an initial value $\theta^{0}=\left(\theta_{1}^{0}, \ldots, \theta_{K}^{0}\right)$.

2.) Given $\theta^{j}$, find the alignment

$$
v_{\theta^{j}}\left(x_{1}, \ldots, x_{n}\right)=\left(v_{1}, \ldots, v_{n}\right)
$$

and partition $x_{1}, \ldots, x_{n}$ into $K$ subsamples, with $x_{k}$ going to the $l^{\text {th }}$ subsample if and only if $v_{k}=l$. Equivalently, define up to $K$ empirical measures

$$
\hat{P}_{l}^{n}\left(A ; \theta^{j}\right):=\frac{\sum_{i=1}^{n} I_{A \times l}\left(x_{i}, v_{i}\right)}{\sum_{i=1}^{n} I_{l}\left(v_{i}\right)}, \quad A \in \mathcal{B}, \quad l \in S .
$$

3.) For every subsample find the MLE given by:

$$
\hat{\mu}_{l}\left(\theta^{j}\right)=\arg \max _{\theta_{l} \in \Theta_{l}} \int \ln f_{l}\left(x ; \theta_{l}\right) \hat{P}_{l}^{n}\left(d x ; \theta^{j}\right),
$$

and take $\theta_{l}^{j+1}=\hat{\mu}_{l}\left(\theta^{j}\right), \quad l \in S$. If for some $l \in S, v_{i} \neq l$ for any $i=1, \ldots, n\left(l^{\text {th }}\right.$ subsample is empty), then the empirical measure $\hat{P}_{l}^{n}$ is formally undefined, in which case we take $\theta_{l}^{j+1}=\theta_{l}^{j}$. We omit this exceptional case in the following discussion. 
VT can be interpreted as follows. Suppose that at step $j, \theta^{j}=\theta^{*}$ and hence $v_{\theta^{j}}$ is obtained using the true parameters. The training is then based on the assumption that the alignment $v\left(x_{1}, \ldots, x_{n}\right)=\left(v_{1}, \ldots, v_{n}\right)$ is correct, i.e., $v_{i}=Y_{i}, i=1, \ldots, n$. In this case, the empirical measures $\hat{P}_{l}^{n}\left(\theta^{j}\right), l \in S$ would be obtained from the i.i.d. sample generated from $P_{l}\left(\theta^{*}\right)$, and the MLE $\hat{\mu}_{l}\left(\theta^{*}\right)$ would be the natural estimator to use. Clearly, under these assumptions $\hat{P}_{l}^{n}\left(\theta^{*}\right) \Rightarrow P_{l}\left(\theta^{*}\right)$ a.s. and, provided that $\left\{f_{l}(\cdot ; \theta): \theta \in \Theta_{l}\right\}$ is a $P_{l}$-Glivenko-Cantelli class and $\Theta_{l}$ is equipped with some suitable metric, $\lim _{n \rightarrow \infty} \hat{\mu}_{l}\left(\theta^{*}\right)=\theta_{l}^{*}$ a.s. Hence, if $n$ is sufficiently large, then $\hat{P}_{l}^{n} \approx P_{l}$ and $\theta_{l}^{j+1}=\hat{\mu}_{l}\left(\theta^{*}\right) \approx \theta_{l}^{*}=\theta_{l}^{j}, \forall l$ i.e. $\theta^{j}=\theta^{*}$ would be (approximately) a fixed point of the training algorithm.

A weak point of the previous argument is that the alignment in general is not correct even when the parameters used to find it are, i.e. generally $v_{i} \neq Y_{i}$. In particular, this implies that the empirical measures $\hat{P}_{l}^{n}\left(\theta^{*}\right)$ are not obtained from an i.i.d. sample taken from $P_{l}\left(\theta^{*}\right)$. Hence, we have no reason to believe that $\hat{P}_{l}^{n}\left(\theta^{*}\right) \Rightarrow P_{l}\left(\theta^{*}\right)$ a.s. and $\lim _{n \rightarrow \infty} \hat{\mu}_{l}\left(\theta^{*}\right)=\theta_{l}^{*}$ a.s. Moreover, we do not even know whether the sequences of empirical measures $\left\{\hat{P}_{l}^{n}\left(\theta^{*}\right\}\right.$ and MLE estimators $\left\{\hat{\mu}_{l}\left(\theta^{*}\right)\right\}$ converge (a.s.) at all.

In [10], we prove the existence of limiting probability measures $Q_{l}\left(\theta, \theta^{*}\right), l \in S$, that depend on $\theta$, the parameters used to find the alignment $v_{\theta}\left(x_{1}, \ldots, x_{n}\right)$, and on $\theta^{*}$, the true parameters with which the random samples are generated. Namely, these $Q_{l}, l \in S$ will be such that for every $l$

$$
\hat{P}_{l}^{n}\left(\theta^{*}\right) \Rightarrow Q_{l}\left(\theta^{*}, \theta^{*}\right), \quad \text { a.s.. }
$$

Suppose also that the parameter space $\Theta_{l}$ is equipped with some metric. Then, under certain consistency assumptions on classes $\mathcal{F}_{l}=\left\{f_{l}\left(\theta_{l}\right): \theta_{l} \in \Theta_{l}\right\}$, the convergence

$$
\lim _{n \rightarrow \infty} \hat{\mu}_{l}\left(\theta^{*}\right)=\mu_{l}\left(\theta^{*}, \theta^{*}\right) \quad \text { a.s. }
$$

can be deduced from (5), where

$$
\mu_{l}\left(\theta, \theta^{*}\right) \stackrel{\text { def }}{=} \arg \max _{\theta_{l}^{\prime} \in \Theta_{l}} \int \ln f_{l}\left(x ; \theta_{l}^{\prime}\right) Q_{l}\left(d x ; \theta, \theta^{*}\right)
$$

We also show that in general, for the baseline Viterbi training $Q_{l}\left(\theta^{*}, \theta^{*}\right) \neq P_{l}\left(\theta^{*}\right)$, implying $\mu_{l}\left(\theta^{*}, \theta^{*}\right) \neq$ $\theta_{l}^{*}$. In an attempt to reduce the bias $\theta_{l}^{*}-\mu_{l}\left(\theta^{*}, \theta^{*}\right)$, we next propose the adjusted Viterbi training. Suppose (5) and (6) hold. Based on (4), we now consider the mapping

$$
\mu_{l}(\theta)=\mu_{l}(\theta, \theta), \quad l=1, \ldots, K
$$

Since this function is independent of the sample, we can define the following correction for the bias:

$$
\Delta_{l}(\theta)=\theta_{l}-\mu_{l}(\theta), \quad l=1, \ldots, K
$$

\section{$\underline{\text { VA1 - Adjusted Viterbi Training }}$}

1.) Choose an initial value $\theta^{0}=\left(\theta_{1}^{0}, \ldots, \theta_{K}^{0}\right)$.

2.) Given $\theta^{j}$, perform the alignment and define $K$ empirical measures $\hat{P}_{l}^{n}\left(\theta^{j}\right)$ as in (3).

3.) For every $\hat{P}_{l}^{n}$, find $\hat{\mu}_{l}\left(\theta^{j}\right)$ as in (雨) and for each $l$, define $\theta_{l}^{j+1}=\hat{\mu}_{l}\left(\theta^{j}\right)+\Delta_{l}\left(\theta^{j}\right)$, where $\Delta_{l}$ is defined $\forall l \in S$ in $(9)$.

Note that, as desired, for $n$ sufficiently large, the adjusted training algorithm has $\theta^{*}$ as its (approximately) fixed point: Indeed, suppose $\theta^{j}=\theta^{*}$. From $(6), \hat{\mu}_{l}\left(\theta^{j}\right)=\hat{\mu}_{l}\left(\theta^{*}\right) \approx \mu_{l}\left(\theta^{*}\right)=\mu_{l}\left(\theta^{j}\right)$, for all $l \in S$. Hence,

$$
\theta_{l}^{j+1}=\hat{\mu}_{l}\left(\theta^{*}\right)+\Delta_{l}\left(\theta^{*}\right) \approx \mu_{l}\left(\theta^{*}, \theta^{*}\right)+\Delta_{l}\left(\theta^{*}\right)=\theta_{l}^{*}=\theta^{j}, \quad l \in S .
$$




\section{Mixture model}

In general, no closed form for the distribution $Q_{l}\left(\theta^{*}, \theta^{*}\right)$ in (5) is available. Therefore, the mapping (8) may be impossible to determine exactly and approximations of $Q_{l}$ should be used for the adjustments of Viterbi training (\$2). However, in the case of the mixture model, the distributions $Q_{l}$ are straightforward to find and the adjusted Viterbi training can therefore be fully specified. In this model, $Y$, the underlying Markov chain, is a sequence of i.i.d. discrete random variables with the state space $S=\{1, \ldots, K\}$ of mixture components. Thus, the transition probabilities are $p_{i j}=p_{j}, i, j \in S$, where $p_{j}$ are mixture weights. To each component $l \in S$ there corresponds a probability distribution $P_{l}$ with density $f_{l}=f_{l}\left(\cdot ; \theta_{l}^{*}\right)$, where $\theta_{i}^{*}$ are the true parameters. Unless explicitly stated otherwise, the mixture weights $p_{l}$ will be assumed to be known. Such a model produces observations $x_{1}, \ldots, x_{n}$, that are regarded as an i.i.d. sample from the mixture distribution $P$ with density

$$
\sum_{i=1}^{K} p_{i} f_{i}=\sum_{i=1}^{K} p_{i} f_{i}\left(\cdot ; \theta_{i}^{*}\right)=f\left(\cdot ; \theta^{*}\right)=f .
$$

For any set of parameters $\theta=\left(\theta_{1}, \ldots \theta_{K}\right)$, the alignment $v_{\theta}$ can be obtained via a Voronoi partition $\mathcal{S}(\theta)=\left\{S_{1}(\theta), \ldots, S_{K}(\theta)\right\}$, where

$$
\begin{aligned}
S_{1}(\theta) & =\left\{x: p_{1} f_{1}\left(x ; \theta_{1}\right) \geq p_{j} f_{j}\left(x ; \theta_{j}\right), \quad \forall j \in S\right\} \\
S_{l}(\theta) & =\left\{x: p_{l} f_{l}\left(x ; \theta_{l}\right) \geq p_{j} f_{j}\left(x ; \theta_{j}\right), \quad \forall j \in S\right\} \backslash\left(S_{1} \cup \ldots \cup S_{l-1}\right), \quad l=2, \ldots, K .
\end{aligned}
$$

Now, the alignment can be defined as follows: $v_{\theta}(x)=l$ if and only if $x \in S_{l}(\theta)$. In particular, given the Voronoi partition $\mathcal{S}(\theta)=\left\{S_{1}, \ldots, S_{l}\right\}$, the empirical measures $\hat{P}_{l}^{n}(3)$ are

$$
\hat{P}_{l}^{n}(A ; \theta)=\frac{\sum_{i=1}^{n} I_{S_{l}(\theta) \cap A}\left(x_{i}\right)}{\sum_{i=1}^{n} I_{S_{l}(\theta)}\left(x_{i}\right)}, \quad A \in \mathcal{B}, \quad l \in S .
$$

Thus, given the same partition, $\hat{\mu}_{l}(\theta)$ (四), the sub-sample MLE for component $l$, becomes

$$
\hat{\mu}_{l}(\theta)=\arg \max _{\theta_{l}^{\prime} \in \Theta_{l}} \int_{S_{l}(\theta)} \ln f_{l}\left(x ; \theta_{l}^{\prime}\right) \hat{P}_{n}(d x),
$$

where $\hat{P}_{n}$ is the ordinary empirical measure associated with the given random sample. The convergence (5) then follows immediately from (14). Indeed, for any $\theta$, by virtue of the Strong Law of Large Numbers we have

$$
\lim _{n \rightarrow \infty} \hat{P}_{l}^{n}(A ; \theta) \stackrel{\text { a.s. }}{=} \frac{P\left(A \cap S_{l}(\theta) ; \theta^{*}\right)}{P\left(S_{l}(\theta) ; \theta^{*}\right)}=\frac{\int_{S_{l}(\theta) \cap A} f\left(x ; \theta^{*}\right) d \lambda(x)}{\int_{S_{l}(\theta)} f\left(x ; \theta^{*}\right) d \lambda(x)}=\frac{\sum_{i} p_{i} \int_{S_{l}(\theta) \cap A} f_{i}\left(x ; \theta_{i}^{*}\right) d \lambda(x)}{\sum_{i} p_{i} \int_{S_{l}(\theta)} f_{i}\left(x ; \theta_{i}^{*}\right) d \lambda(x)} .
$$

Since $\mathcal{X}$ is separable, it follows that $\hat{P}_{l}^{n} \Rightarrow Q_{l}$ a.s., where

$$
q_{l}\left(x ; \theta, \theta^{*}\right) \propto f\left(x ; \theta^{*}\right) I_{S_{l}(\theta)}=\left(\sum_{i} p_{i} f_{i}\left(x ; \theta^{*}\right)\right) I_{S_{l}(\theta)}, \quad l=1, \ldots, K
$$

are the densities of respective $Q_{l}\left(\theta, \theta^{*}\right)$ 's.

Now it is clear that even when the partition $\mathcal{S}\left(\theta^{*}\right)$ is obtained using the true parameters $\theta^{*}, Q_{l}\left(\theta^{*}, \theta^{*}\right)$, the limiting distribution (density $q_{l}\left(x ; \theta^{*}, \theta^{*}\right)$ ), can be different from $P_{l}\left(\theta^{*}\right)$, the desired distribution (density $f_{l}\left(x ; \theta^{*}\right)$ ). Likewise, $\mu_{l}\left(\theta^{*}\right)(8)$ can be different from

$$
\theta_{l}^{*}=\arg \max _{\theta_{l}^{\prime} \in \Theta_{l}} \int \ln f_{l}\left(x ; \theta_{l}^{\prime}\right) f_{l}\left(x ; \theta_{l}^{*}\right) d \lambda(x) .
$$


In order to this, note that (7) and (8) in the context of the mixture model specialize to

$$
\begin{aligned}
\mu_{l}\left(\theta, \theta^{*}\right) & =\arg \max _{\theta_{l}^{\prime} \in \Theta_{l}} \int_{S_{l}(\theta)} \ln f_{l}\left(x ; \theta_{l}^{\prime}\right) f\left(x ; \theta^{*}\right) d \lambda(x) \\
\mu_{l}(\theta) & =\arg \max _{\theta_{l}^{\prime} \in \Theta_{l}} \int_{S_{l}(\theta)} \ln f_{l}\left(x ; \theta_{l}^{\prime}\right)\left(\sum_{i} p_{i} f_{i}\left(x ; \theta_{i}\right)\right) d \lambda(x),
\end{aligned}
$$

respectively. We also emphasize that $\Delta$ can be significant which justifies the adjustment.

Example 3.1 Let

$$
f\left(x ; \theta^{*}\right)=\frac{1}{K} \sum_{l=1}^{K} \phi\left(x ; \theta_{l}^{*}\right),
$$

where $\phi\left(x ; \theta_{l}^{*}\right)$ is the density of the d-variate normal distribution with identity covariance matrix and vector of means $\theta_{l}^{*} \in \mathbb{R}^{d}=\Theta_{l}$ for $l=1,2, \ldots, K$. In this case, for each $K$-tuple of parameters $\theta=\left(\theta_{1}, \ldots, \theta_{K}\right)$, the decision-rule for the alignment is essentially as follows (disregarding possible ties): $v_{\theta}(x)=i$ if and only if $\left\|x-\theta_{i}\right\| \leq \min _{j}\left\|x-\theta_{j}\right\|$. Thus, the decision regions in this case correspond to the Voronoi partition in its original sense, justifying our generalization of this term. Now, it can be easily seen that for all $m=1, \ldots, d$ :

$$
\left(\mu_{l}(\theta)\right)_{m}=\frac{\sum_{i=1}^{K} \int_{S_{l}(\theta)} x_{m} \phi\left(x ; \theta_{i}\right) d x_{1} \cdots d x_{d}}{\sum_{i=1}^{K} \int_{S_{l}(\theta)} \phi\left(x ; \theta_{i}\right) d x_{1} \cdots d x_{d}} .
$$

When $d$ and $K$ are large, the exact integration in (18) still requires intensive computations, for which reason one may be interested in approximations of (18). In the context of the above example, one might think of the following approximations for $\Delta_{l}(\theta)=\theta_{l}-\mu_{l}(\theta)$ :

1.) Approximate $\left(\sum_{l} f_{i}\left(x ; \theta_{i}\right)\right) I_{S_{l}(\theta)}$ in $(18)$ by $f_{l}\left(\theta_{l}, x\right) I_{S_{l}(\theta)}$, so

$$
\left(\mu_{l}\right)_{m} \approx \frac{\int_{S_{l}(\theta)} x_{m} \phi\left(x ; \theta_{l}\right) d x_{1} \cdots d x_{d}}{\int_{S_{l}(\theta)} \phi\left(x ; \theta_{l}\right) d x_{1} \cdots d x_{d}} .
$$

This approximation is based on the limiting case when the components are "infinitely" far from each other.

2.) If $K>d$, then some components are fully surrounded by others, namely, the partition cells corresponding to such "internal" components are bounded (Figure 1). It is then conceivable that $\Delta_{l}$ 's that correspond to the bounded cells are less significant than the others, in which case one might only correct the estimators of the internal components.

3.) Every Voronoi cell is determined by several hyperplanes and every such hyperplane $H^{j}$ gives rise to $\Delta_{l}^{j}$, a component of $\Delta_{l}$ in the direction perpendicular to $H^{j}$ and corresponding to the $l^{\text {th }}$ term in the sum in (18). Thus, $\Delta_{l}=\sum_{j} \Delta_{l}^{j}$ (see, for example, the $l=1$ cell in Figure 1). It may be reasonable to find only the "main direction" of correction, i.e. the largest $\Delta_{l}^{j}$ for each $l$ (see, for example, the $l=2$ cell in Figure 1).

Remark 3.2 In Example 3.1, the decision regions correspond to the Voronoi partition in its original sense. Moreover, it is easy to see that in this particular case, the Viterbi training is none other than the well-known (generalized) Lloyd algorithm designed for finding vector quantizers, which in this case are also called $K$-means (see, e.g. [21]). In this case, the estimators obtained by the Viterbi training are empirical $K$-means. The latter estimators enjoy certain desirable properties, and in particular they are consistent with respect to the population $K$-means [17]. However, they need not be consistent with respect to $\theta^{*}$, our parameters of interest. In the mixture case, the Viterbi training can always be considered as the (generalized) Lloyd algorithm, and the estimators obtained by Viterbi training can be regarded as (generalized) empirical K-means [37. This observation links the study of Viterbi Training and related algorithms to the theory of vector quantization. 


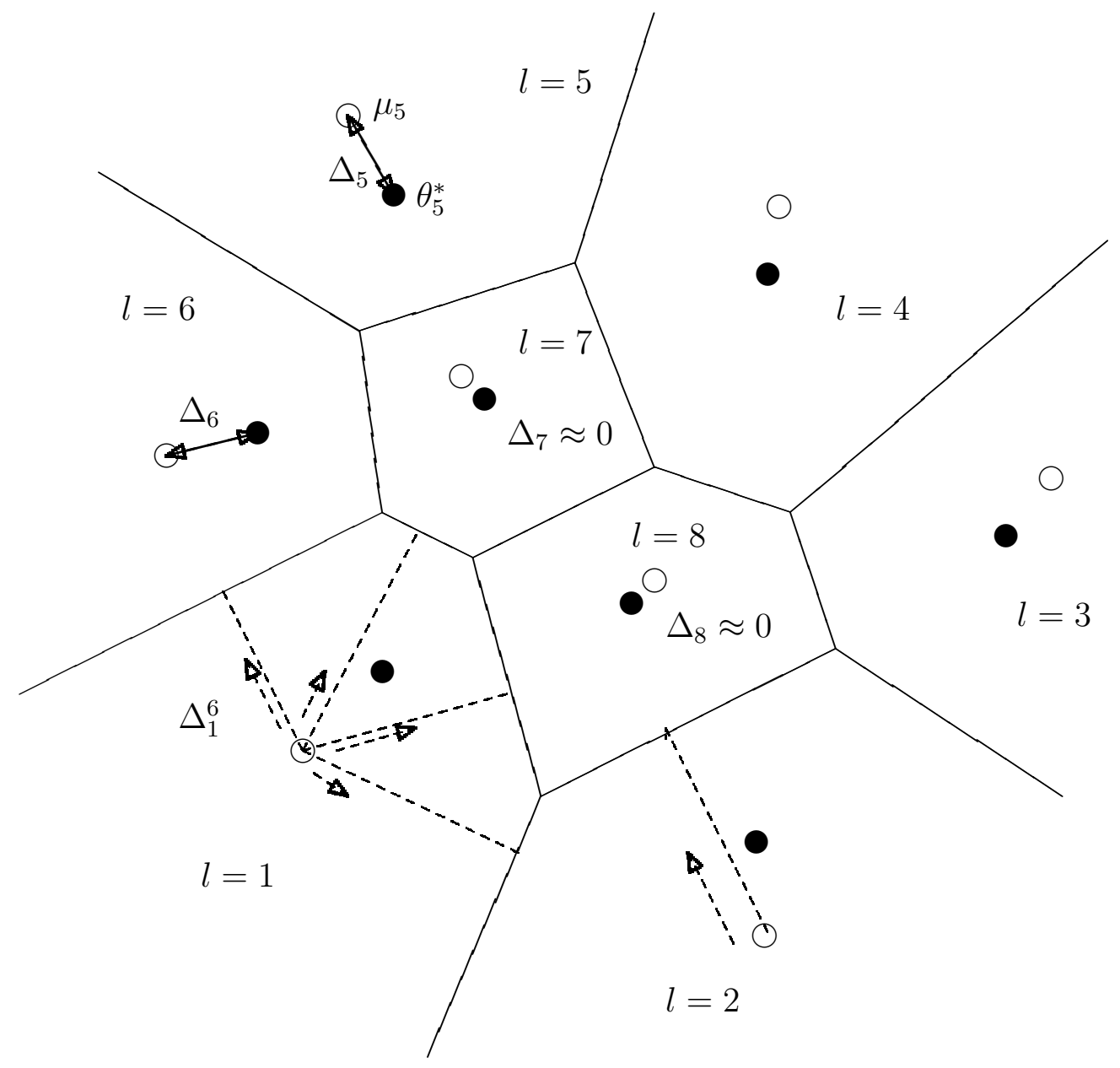

Figure 1: An example of the Voronoi partition for multiple components. True parameters $\theta^{*}$ and corresponding $\mu\left(\theta^{*}\right)$ are marked with solid and transparent dots, respectively. For $l=1$ component, $\Delta_{l}^{j}\left(\theta^{*}\right)$, the correction components, are indicated. For $l=2$ component, the main direction of the correction is indicated. It appears a reasonable approximation to neglect the corrections for the estimators corresponding to the bounded Voronoi regions. 


\section{VA2 - Adjustment of the second type}

The adjusted Viterbi training is designed to asymptotically fix the true parameter $\theta^{*}$, returning approximately the correct solution given this solution as the initial guess and given an infinitely large data sample: $\operatorname{VA} 1\left(\theta^{*}\right) \approx \theta^{*}$. VA2 goes further and attempts to maximally expand $\left\{\theta: \operatorname{VA} 1(\theta) \approx \theta^{*}\right\}$, the set of parameter values that are asymptotically mapped to the true ones, to $\left\{\theta: \operatorname{VA} 2(\theta) \approx \theta^{*}\right\}$. Specifically, if the algorithm ever arrives at $\mathcal{S}\left(\theta^{*}\right)$, the Voronoi partition corresponding to the true parameters $\theta^{*}$, then we would like to coerce the adjusted estimates to return $\theta^{*}$. Let us explain these ideas in more detail.

Let $\mathcal{S}^{*}$ stand for $\mathcal{S}\left(\theta^{*}\right)$, the true Voronoi partition (that also coincides with the Bayes decision boundary). The mapping $\theta \mapsto \mathcal{S}(\theta)$ is generally many-to-one, hence the set $\Theta\left(\mathcal{S}^{*}\right)=\left\{\theta: \mathcal{S}(\theta)=\mathcal{S}^{*}\right\}$ generally contains more than one element. (This also means that guessing $\mathcal{S}^{*}$, i.e. guessing any element from $\Theta\left(\mathcal{S}^{*}\right)$, is generally easier than guessing $\theta^{*}$.) We now introduce VA2:

Note first that $\mu_{l}\left(\theta, \theta^{*}\right)$ in (16), as well as the estimate $\hat{\mu}_{l}(\theta)$ in (15), depends on $\theta$ through $\mathcal{S}(\theta)$ only. However, the correction $\Delta_{l}(\theta)=\theta_{l}-\mu_{l}(\theta, \theta)$ does depend on $\theta$ fully and hence would not generally work (in the sense of (10)) for an arbitrary $\theta^{j} \in \Theta\left(\mathcal{S}^{*}\right)$ unless $\theta^{j}=\theta^{*}$. We now attempt to improve the first type of adjustment that is based on adding $\Delta\left(\theta^{j}\right)$ to $\hat{\mu}\left(\theta^{j}\right)$. Namely, we propose the following iterative update for $l=1, \ldots, K$ : Next, define $\mu_{l, \Theta\left(\mathcal{S}\left(\theta^{0}\right)\right)}(\theta)$ (as function of $\theta$ only) to be the restriction of $\mu_{l}\left(\theta^{0}, \theta\right)$ to $\Theta\left(\mathcal{S}\left(\theta^{0}\right)\right)$, and write $\mu_{l, \theta^{0}}(\theta)$ in place of the more cumbersome $\mu_{l, \Theta\left(\mathcal{S}\left(\theta^{0}\right)\right)}(\theta)$. Let

$$
\theta_{l}^{j+1}=\left\{\begin{array}{l}
\mu_{l, \theta^{j}}^{-1}\left(\hat{\mu}_{l}\left(\theta^{j}\right)\right), \quad \text { if a unique } \mu_{l, \theta^{j}}^{-1}\left(\hat{\mu}_{l}\left(\theta^{j}\right)\right) \text { exists } \\
\hat{\mu}_{l}\left(\theta^{j}\right)+\Delta_{l}\left(\theta^{j}\right) \quad \text { otherwise. }
\end{array}\right.
$$

For any $\theta^{j}$ and $\theta^{*}$, the event that $\hat{\mu}\left(\theta^{j}, \theta^{*}\right)$ belongs to the range of $\mu\left(\theta^{j}, \theta\right)$ as a function of $\theta \in \Theta\left(\mathcal{S}\left(\theta^{j}\right)\right)$ is of zero probability, as Example 4.1 illustrates. Hence, the introduction of the individual inverses $\mu_{l, \theta^{j}}^{-1}$ $l=1, \ldots, K$ is essential, although still not always effective: In some mixture models (a mixture of normal distributions with unequal weights is one such example), for a fixed $l$, the event that $\hat{\mu}_{l}\left(\theta^{j}\right)$ belongs in the range of $\mu_{l}\left(\theta^{j}, \theta\right)$ (as function of $\theta \in \Theta\left(\mathcal{S}\left(\theta^{j}\right)\right)$ ) need not occur with probability one for all $\theta^{j}$ and $\theta^{*}$. This, and also the fact that the inverses in general need not have a closed form, or may require intensive computations, may reduce the attractiveness of the suggested method. Further discussion of the computational issues related to this method is outside the scope of this paper, except for mentioning the possibility of various, e.g. linear or quadratic, approximations of the above functions $\mu_{l, \theta}^{-1}$.

In order to better understand the meaning of the new adjustment, imagine that $\theta^{j} \in \Theta\left(\mathcal{S}^{*}\right)$. We would then expect for $l=1, \ldots, K$ :

$$
\theta_{l}^{j+1}=\mu_{l, \theta^{j}}^{-1}\left(\hat{\mu}_{l}\left(\theta^{j}\right)\right)=\mu_{l, \theta^{*}}^{-1}\left(\hat{\mu}_{l}\left(\theta^{*}\right)\right) \approx \mu_{l, \theta^{*}}^{-1}\left(\mu_{l}\left(\theta^{*}, \theta^{*}\right)\right)=\theta_{l}^{*} .
$$

The above argument, of course, also depends on the regularity of the above inverses at $\mu_{l}\left(\theta^{*}, \theta^{*}\right) l=$ $1, \ldots, K$, and in this regard our experiments in 8 国 provide encouraging results for an important model similar to the model in the following example:

Example 4.1 Let $f\left(x ; \theta^{*}\right)=\frac{1}{2} \phi\left(x-\theta_{1}^{*}\right)+\frac{1}{2} \phi\left(x-\theta_{2}^{*}\right)$, where $\phi$ is the density of the standard normal distribution. In this case any Voronoi partition is specified by a single parameter $t=0.5\left(\theta_{1}+\theta_{2}\right)$ solving $\phi\left(t-\theta_{1}\right)=\phi\left(t-\theta_{2}\right)$ (ties are evidently inessential in this context). The true Voronoi partition corresponds to $t^{*}=0.5\left(\theta_{1}^{*}+\theta_{2}^{*}\right)$. Given a Voronoi partition $\mathcal{S}(t(\theta)), \Theta(t)=\left\{(t-a),(t+a): a \in \mathbb{R}^{+}\right\}$. Hence, restricted to $\Theta(t)$, the function $\mu_{\mathcal{S}(t)}(\theta)=\left(\mu_{1, \mathcal{S}(t)}(\theta), \mu_{2, \mathcal{S}(t)}(\theta)\right)$ depends on one parameter only: Let a be this parameter and define $\mu_{\mathcal{S}(t)}(\theta(a))=\left(\mu_{1}(a), \mu_{2}(a)\right)$ as follows: $\mu_{1}(a)=-a(1-2 \Phi(-a))-2 \phi(-a)+t$, $\mu_{2}(a)=2 t-\mu_{1}(a)$, where $\Phi$ is the distribution function of the standard normal distribution. After calculating $\hat{\mu}_{1}<\hat{\mu}_{2}$ from the data, the inversion equations of (20) become

$$
t-[a(1-2 \Phi(-a))+2 \phi(a)]=\hat{\mu}_{1}, \quad t+[a(1-2 \Phi(-a))+2 \phi(a)]=\hat{\mu}_{2} .
$$

Obviously (21) has a (unique) solution if and only if $\hat{\mu}_{1}, \hat{\mu}_{2}$ are symmetric with respect to $t$ and the probability of this latter event is clearly zero under the model. Thus, as suggested in (20), we consider 
the equations separately:

$$
\begin{aligned}
& a(1-2 \Phi(-a))+2 \phi(a)=t-\hat{\mu}_{1} \\
& a(1-2 \Phi(-a))+2 \phi(a)=\hat{\mu}_{2}-t .
\end{aligned}
$$

It can be shown that (22) and (23) have unique solutions, let us denote the latter by $a_{1}$ and $a_{2}$, respectively. The points $t-a_{1}$ and $t+a_{2}$ will be now taken as the estimators of $\theta_{1}^{*}$ and $\theta_{2}^{*}$ for the next step of iterations.

\section{$\underline{\mathrm{VA} 2}$}

1.) Choose $\theta^{0}=\left(\theta_{1}^{0}, \ldots, \theta_{K}^{0}\right)$.

2.) Given $\theta^{j}$, find $\mathcal{S}\left(\theta^{j}\right)$ and define empirical measures $\hat{P}_{l}^{n}\left(\theta^{j}\right)$ as in (14).

3.) For every $\hat{P}_{l}^{n}$, find $\hat{\mu}_{l}\left(\theta^{j}, \theta^{*}\right)$ as in (15).

4.) Update $\theta^{j+1}$ in accordance with (20).

\subsection{Unknown weights}

We consider the case when the mixture weights $p_{l}$ are unknown, which corresponds to the case of the unknown transition parameters $(P, \pi)$ in the general HMM context.

The Voronoi partition depends on the weight-vector $p=\left(p_{1}, \ldots, p_{K}\right)$ as well as on $\theta$. Hence, $\mathcal{S}(\theta, p)$ and the vector $p$ should be reestimated at each step along with $\theta$. Given a Voronoi partition $\mathcal{S}=$ $\left\{S_{1}, \ldots, S_{K}\right\}$, the simplest way to estimate the weights $p_{l}$ is to take $p_{l}=\hat{P}_{n}\left(S_{l}\right)$, the empirical measure of $S_{l}$. Hence all the algorithms considered so far can be modified accordingly to include the weight estimation as in (24).

$$
p_{l}^{j+1}=\hat{P}_{n}\left(S_{l}\left(\theta^{j}, p^{j}\right)\right), \quad l=1, \ldots, K .
$$

Taking into account the asymptotics, it is easy to correct the estimators $p^{j+1}$ as well. Indeed, suppose $\theta^{j}=\theta^{*}$ and $p^{j}=p$, i.e. $\mathcal{S}\left(\theta^{j}, p^{j}\right)=\mathcal{S}\left(\theta^{*}, p\right)=\mathcal{S}^{*}$. If $n \rightarrow \infty$, then

$$
\hat{P}_{n}\left(S_{l}\left(\theta^{*}, p\right)\right) \stackrel{\text { a.s. }}{\rightarrow} P\left(S_{l}\left(\theta^{*}, p\right)\right)=\int_{S_{l}\left(\theta^{*}, p\right)} f\left(x ; \theta^{*}\right) d \lambda=\sum_{i} p_{i} \int_{S_{l}\left(\theta^{*}, p\right)} f_{i}\left(x ; \theta_{i}^{*}\right) d \lambda .
$$

In general the latter differs from $p_{l}$. The difference is $p_{l}-P\left(S_{l}\left(\theta^{*}, p\right)\right)$. Hence, by analogy with (9), we can define the weight correction $D(\theta, p)=\left(D_{1}(\theta, p), \ldots, D_{K}(\theta, p)\right)$ as follows:

$$
D_{l}(\theta, p)=p_{l}-\sum_{i} p_{i} \int_{S_{l}(\theta, p)} f_{i}\left(x ; \theta_{i}\right) d \lambda,
$$

which is also data independent. We now summarize the above by giving a formal definition of the adjusted Viterbi training with the weight correction. The Viterbi training and the second type of adjusted Viterbi training with $p$ unknown can be defined similarly.

\section{$\underline{\text { VA1 with the weight correction }}$}

1.) Choose $\theta^{0}=\left(\theta_{1}^{0}, \ldots, \theta_{K}^{0}\right)$ and $p^{0}=\left(p_{1}^{0}, \ldots p_{K}^{0}\right)$

2.) Given $\theta^{j}=\left(\theta_{1}^{j}, \ldots, \theta_{K}^{j}\right)$ and $p^{j}=\left(p_{1}^{j}, \ldots p_{K}^{j}\right)$ define the Voronoi partition $\mathcal{S}\left(\theta^{j}, p^{j}\right)=\left\{S_{1}, \ldots, S_{K}\right\}$ as in (12) and (13), and the empirical measures $\hat{P}_{l}^{n}\left(\theta^{j}, p^{j}\right)$ as in (14).

3.) Put $\theta^{j+1}=\hat{\mu}^{j}\left(\theta^{j}\right)+\Delta\left(\theta^{j}\right)$, where $\hat{\mu}^{j}$ is defined in (17).

4.) Put $p^{j+1}=\hat{P}_{n}\left(S_{l}\left(\theta^{j}, p^{j}\right)\right)+D\left(\theta^{j}, p^{j}\right)$. 


\section{Simulation studies}

In order to support our theory of adjusted Viterbi Training we simulate 1000 i.i.d. random samples of size 1000 according to the following mixture:

$$
\frac{1}{\sqrt{2 \pi}}\left(p e^{-\frac{\left(x-\theta_{1}\right)^{2}}{2}}+(1-p) e^{-\frac{\left(x-\theta_{2}\right)^{2}}{2}}\right)
$$

The true parameters in our experiments are $\theta^{*}=(-2.5,0)$ and $(p, 1-p)=(0.7,0.3)$. The corresponding density is plotted in Figure 2. Note that for all such mixtures with $p>0.5$ and $\theta_{1}<\theta_{2}$,

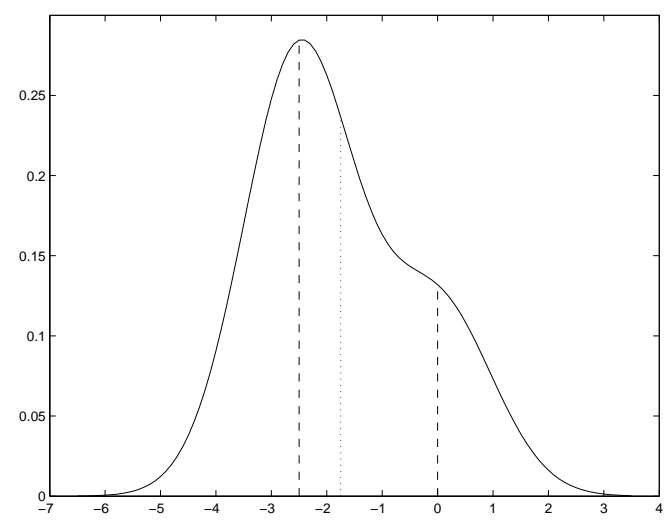

Figure 2: $\frac{1}{\sqrt{2 \pi}}\left(0.7 e^{-\frac{(x+2.5)^{2}}{2}}+0.3 e^{-\frac{x^{2}}{2}}\right)$. The dashed vertical lines indicate the means of the individual components, the dotted line marks the mean of the mixture.

$\theta_{2}-\theta_{1}<\sqrt{2 p /(1-p)}(=2.1602$ in our case $)$ implies that the both means fall on one side of the decision boundary, which makes detection of the second component particularly difficult as is already becoming the case in our setting with $\theta_{2}^{*}-\theta_{1}^{*}=2.5$.

Our main goal is to compare the performances of VT, VA1, and EM in terms of the accuracy, convergence, amount of computations per iteration, and the total amount of computations. We implement these algorithms in Matlab [13], providing a fair comparison of their computational intensities based on their execution times. Our code is available for the reader's perusal [9] and is fully-optimized for speed in the case of VT and EM. Consequently, our simulations possibly only overestimate the execution times for VA1.

Additionally, we compare VA2 with the above algorithms by the accuracy and convergence. We use a numerical solver to compute the adjustment function of VA2 and presently make no effort to replace this by a computationally efficient approximation. Hence, we do not discuss the computational intensity of VA2 in this work.

In our experiments, the algorithms are instructed to terminate as soon as the $L_{2}$ distance between consecutive $\theta$ updates falls below 0.001. We also provide a high precision MLE computed with a built-in matlab optimization function. The cases of known and unknown weights $(\S 4.1)$ are considered in $\S 5.1$ and $\$ 5.2$, respectively. We report the following statistics for each of the algorithms in the form: average \pm one standard deviation.

- $\theta=\left(\theta_{1}, \theta_{2}\right)$ - the estimates of the means;

- $p$ - the estimate of the weight of the first component;

- $\left\|\theta-\theta^{*}\right\|_{1,2}-L_{1^{-}}$and $L_{2}$-normed distances between $\theta$ and the true parameters;

- $n$ - number of steps used by the algorithm; 
- $T$ - total time in milliseconds to execute the entire algorithm;

- $t$ - time in milliseconds to execute one iteration of the algorithm;

\subsection{Known weights}

It is often the case in speech recognition models that the weights are assumed known.

First, consider $(-1,2)$ as an "arbitrary" initial guess for $\theta$. Table 1 presents the performance statistics based on the 1000 samples. The baseline Viterbi method terminates quickly (on average in 9.04 steps), outperformed only by VA2, but is the least accurate among the considered methods. As expected, VT also requires fewest computations: $0.2 \mathrm{~ms}$ per iteration and $1.85 \mathrm{~ms}$ total. Ranked from low to high, accuracies of VA1, VA2, and EM appear similar, and are about three times superior to that of VT. In units of the VT execution time, EM compares to VA1 as 16.85:6.7 per iteration, and as 20.43:7.59 by the total execution times.

In order to illustrate the asymptotic fixed point property, we initialize the algorithms to $(-2.5,0)$, the true value of the parameters. In this case, as expected, the both types of adjustments exit noticeably faster than VT and EM, are comparable in accuracy to EM, and are about three times more accurate than VT. Unlike VA1, VA2 or EM, the baseline algorithm, as predicted, disturbs the correct initial guess, resulting in an appreciable bias. The times per iteration of VA1 and EM are similar to as before, and their total times are (in units of the VT time): 5.71 and 16.03, respectively.

In order to illustrate the idea of the second type of adjustment, we now initialize the algorithms to $(-3.1229,0.8771)$, which produces the same decision boundary $t=-0.9111$ as $\theta^{*}=(-2.5,0)$, the true values. Note that since VT and VA2 depend on the initial guess only via the decision boundary, they produce in this case exactly the same results (disregarding a small rounding error) as in the case of the correct initial guess (Table 2). As expected, VA2 now terminates significantly faster than its competitors, and accuracy-wise is only slightly superior to VA1 and slightly inferior to EM. The times per iteration of VA1 and EM are similar to as before, and their total times are 7.84 and 20.83, respectively.

\subsection{Unknown weights}

Assume now that the weights are unknown $(\S 4.1)$ and hence need to be estimated along with the means. We use the same data and the same three types of conditions as in the case of known weights: Arbitrary initialization to $(-1,2)$ (Table 4), initialization to the correct values $(-2.5,0)$ (Table 5 ), and initialization to $(-3.1229,0.8771)$, an arbitrary point giving rise the correct intercomponent boundary (Table 6). VT and the adjusted algorithms VA1 and VA2 in this case are implemented with the asymptotic correction (26). (The maximization in the high precision MLE is now performed in the three variables.)

The adjusted algorithms now converge 1.7 (VA1) and 2 (VA2) times faster than EM, and, what is more remarkable, VA1 and VA2 converge even faster than VT. The per iteration times of VA1 and EM compare as about 1.8:4.8 for all the initializations, and the total times - as 1.8:8.7 (arbitrary guess), 1.3:6.67 (true values), and 1.73:7.64 (true boundary), all in units of the VT time. VA1 and VA2 are again at least three times more accurate than VT in $\theta$ estimation and about one standard deviation more accurate than VT in the weight estimation. They are also comparable in accuracy to EM.

\subsection{Summary of the results}

VA1 is consistently close in accuracy to EM which is always superior to Viterbi Training: Specifically, in estimating the means, the gain in accuracy is about three-fold as measured by $L_{1}$ - and $L_{2}$-distances, and in estimating the weights, it is about one standard deviation.

VA1 always converges almost as fast as VT and noticeably (by $30 \%$ in the case of unknown weights) faster than EM.

When the weights are known, an iteration of VA1 is about six times longer than that of VT and is more than twice as fast as that of EM. By total execution, VA1 is at most eight times slower than VT and is more than two and a half times faster than EM. 
When the weights are unknown, VA1 is at most twice slower than VT and more than two and a half times faster than EM, per iteration. It is also about $50 \%$ slower than than VT and more than four times faster than EM in total times.

Accuracy of VA2 is consistently between those of VA1 and EM, and VA2 additionally converges faster than VA1.

\section{Conclusion}

We have considered the problem of parameter estimation of the emission distribution in Hidden Markov Models in connection with the two most common estimation algorithms: the EM algorithm and the Viterbi Training algorithm. We have identified the sources of bias, or lack of consistency, in VT estimation in comparison with EM (MLE) estimation. In the case of HMM, EM computes the MLE, which is often consistent. Trading the EM's accuracy for the VT's ease of computations, one loses, among other things, the asymptotic fixed point property: VT no longer holds the true parameter values fixed, even asymptotically. In this work, we have restored this property, and consequently recovered a certain amount of the EM's accuracy, without a significant increase in computations relative to Viterbi Training. Specifically, we have derived two types of adjustments to the baseline Viterbi Training algorithm. Our first algorithm, VA1, that we also present as the central contribution of this work, is a modification of VT that restores the asymptotic fixed point property. We also present evidence that, at least in the case of mixture models (a special and important case of HMM), the price in extra computations for this increase in accuracy can be made reasonable. The second algorithm, VA2, in addition to restoring the fixed point property, also ensures that the true parameters are asymptotically found as soon as the true alignment (i.e. Voronoi partition) has been found. This latter feature may require intensive computations, undermining Viterbi Training as a computationally feasible alternative to EM. We intend to investigate feasible approximations to the correction function of VA2 in future work.

Certainly, the final decision as to which algorithm to use is application dependent, and this work presents valuable information to facilitate such selection, especially in the context of the Gaussian mixture models. For this special case, we have provided simulation studies based on 1000 large random samples which illustrate the key features of the adjusted algorithms in contrast with EM and baseline Viterbi Training. In our simulations, VA1 demonstrates a significant increase of accuracy (three-fold and one standard deviation in in estimating the mixture means and weights, respectively) relative to VT. In fact, the accuracy of VA1 is already comparable to that of EM. Computation-wise, VA1 in our studies is still several factors faster than EM. We therefore suggest replacing VT by VA1 in applications that can afford computing (to a variable precision) the correction function in appreciation of the increased accuracy.

\section{References}

[1] L. Baum and T. Petrie. Statistical inference for probabilistic functions of finite state Markov chains. Ann. Math. Stat., 37:1554-1563, 1966.

[2] J. Bilmes. A gentle tutorial of the EM algorithm and its application to parameter estimation for Gaussian mixture and hidden Markov models. Technical Report 97-021, International Computer Science Institute, 1998.

[3] P. Chou, T. Lookbaugh, and R. Gray. Entropy-constrained Vector Quantization. IEEE Transaction on Acoustic Speech and Signal Processing, 37(1), 1989.

[4] G. Ehret, P. Reichenbach, U. Schindler, C. Horvath, S. Fritz, M. Nabholz, and P. Bucher. DNA binding specificity of different STAT proteins. The Journal of Biological Chemistry, 276(9):66756688, 2001.

[5] R. Gray, T. Linder, and J. Li. A lagrangian formulation of zador's entropy-constrained quantization theorem. IEEE Transactions on Information Theory, 48(3), 2000. 


\begin{tabular}{|c|c|c|c|c|c|}
\hline & VT & VA1 & VA2 & EM & MEL \\
\hline$\theta_{1}$ & $-2.4869 \pm 0.0497$ & $-2.4952 \pm 0.0500$ & $-2.4959 \pm 0.0498$ & $-2.4970 \pm 0.0456$ & $-2.4973 \pm 0.0456$ \\
\hline$\theta_{2}$ & $0.2880 \pm 0.0732$ & $0.0099 \pm 0.0917$ & $0.0082 \pm 0.0916$ & $0.0030 \pm 0.0757$ & $0.0024 \pm 0.0757$ \\
\hline$\left\|\theta-\theta^{*}\right\|_{1}$ & $0.3291 \pm 0.0844$ & $0.1138 \pm 0.0681$ & $0.1133 \pm 0.0678$ & $0.0958 \pm 0.0562$ & $0.0958 \pm 0.0562$ \\
\hline$\left\|\theta-\theta^{*}\right\|_{2}$ & $0.2927 \pm 0.0727$ & $0.0902 \pm 0.0537$ & $0.0899 \pm 0.0536$ & $0.0761 \pm 0.0451$ & $0.0761 \pm 0.0451$ \\
\hline$n$ & $9.04 \pm 1.55$ & $10.49 \pm 1.61$ & $7.84 \pm 1.59$ & $11.20 \pm 0.42$ & $\mathrm{~N} / \mathrm{A}$ \\
\hline$t$ & $0.20 \pm 0.05$ & $1.34 \pm 0.19$ & $39.24 \pm 1.56$ & $3.37 \pm 0.07$ & $\mathrm{~N} / \mathrm{A}$ \\
\hline$T$ & $1.85 \pm 0.55$ & $14.04 \pm 2.95$ & $308.57 \pm 68.63$ & $37.79 \pm 1.56$ & $\mathrm{~N} / \mathrm{A}$ \\
\hline
\end{tabular}

Table 1: "Arbitrary" initial guess.

\begin{tabular}{|c|c|c|c|c|c|}
\hline & VT & VA1 & VA2 & EM & MLE \\
\hline$\theta_{1}$ & $-2.4904 \pm 0.0495$ & $-2.4973 \pm 0.0488$ & $-2.4973 \pm 0.0490$ & $-2.4973 \pm 0.0455$ & $-2.4973 \pm 0.0456$ \\
\hline$\theta_{2}$ & $0.2820 \pm 0.0729$ & $0.0051 \pm 0.0880$ & $0.0052 \pm 0.0892$ & $0.0024 \pm 0.0753$ & $0.0024 \pm 0.0757$ \\
\hline$\left\|\theta-\theta^{*}\right\|_{1}$ & $0.3223 \pm 0.0829$ & $0.1087 \pm 0.0661$ & $0.1102 \pm 0.0664$ & $0.0953 \pm 0.0561$ & $0.0958 \pm 0.0562$ \\
\hline$\left\|\theta-\theta^{*}\right\|_{2}$ & $0.2867 \pm 0.0721$ & $0.0861 \pm 0.0523$ & $0.0874 \pm 0.0525$ & $0.0756 \pm 0.0450$ & $0.0761 \pm 0.0451$ \\
\hline$n$ & $5.56 \pm 1.72$ & $5.06 \pm 1.57$ & $4.73 \pm 1.55$ & $5.69 \pm 1.29$ & N/A \\
\hline$t$ & $0.22 \pm 0.02$ & $1.37 \pm 0.05$ & $42.23 \pm 0.94$ & $3.42 \pm 0.08$ & N/A \\
\hline$T$ & $1.21 \pm 0.31$ & $6.91 \pm 2.05$ & $199.52 \pm 65.67$ & $19.39 \pm 4.28$ & N/A \\
\hline
\end{tabular}

Table 2: Correct initial guess.

[6] X. Huang, Y. Ariki, and M. Jack. Hidden Markov models for speech recognition. Edinburgh University Press, 1990.

[7] F. Jelinek. Statistical methods for speech recognition. The MIT Press, 2001.

[8] B. Juang and L. Rabiner. The segmential k-means training procedure for estimating parameters of hidden Markov models. IEEE Trans. Acoustics, Speech, Signal Proc., 38(9):1639-1641.

[9] A. Koloydenko and J. Lember. Matlab code for simulation studies of adjusted Viterbi training. Available at: http://euridice.tue.nl/ akoloide/VA/, 102003.

[10] J. Lember and A. Koloydenko. The Theory of Adjusted Viterbi Training. Technical Report 019, Eurandom, P.O. Box 513 - 5600 MB Eindhoven, The Netherlands, December 2003. in preparation.

[11] J. Li and R. Gray. Context-based multiscale classification of document images using wavelet coefficient distributions. IEEE Transactions on Image Processing, 9(9), 2000.

[12] J. Li, R. Gray, and R. Olshen. Multiresolution image classification by hierarchical modeling with two dimensional hidden Markov models. IEEE Transactions on Information Theory, 46(5), 2000.

[13] The MathWorks, Inc., http://www.mathworks.com/access/helpdesk/help/helpdesk.shtm. Getting Started with Matlab.

[14] H. Ney, V. Steinbiss, R. Haeb-Umbach, B. Tran, and U. Essen. An overview of the Philips research system for large vocabulary continuous speech recognition. International Journal of Pattern Recognition and Artificial Intelligence, 8(1):33-70, 1994.

[15] F. Och and H. Ney. Improved statistical alignment models. In Proceedings of the 38th Annual Meeting of the Association for Computational Linguistics, http://acl.ldc.upenn.edu/P/P00/P00-1056.pdt, 2000.

[16] U. Ohler, H. Niemann, G. Liao, and G. Rubin. Joint modeling of DNA sequence and physical properties to improve eukaryotic promoter recognition. Bioinformatics, 17(Suppl. 1):S199-S206, 2001. 


\begin{tabular}{|c|c|c|c|c|c|}
\hline & VT & VA1 & VA2 & EM & MLE \\
\hline$\theta_{1}$ & $-2.4904 \pm 0.0495$ & $-2.4954 \pm 0.0497$ & $-2.4973 \pm 0.0490$ & $-2.4971 \pm 0.0456$ & $-2.4973 \pm 0.0456$ \\
\hline$\theta_{2}$ & $0.2820 \pm 0.0729$ & $0.0094 \pm 0.0909$ & $0.0052 \pm 0.0892$ & $0.0030 \pm 0.0757$ & $0.0024 \pm 0.0757$ \\
\hline$\left\|\theta-\theta^{*}\right\|_{1}$ & $0.3223 \pm 0.0829$ & $0.1131 \pm 0.0668$ & $0.1102 \pm 0.0664$ & $0.0958 \pm 0.0562$ & $0.0958 \pm 0.0562$ \\
\hline$\left\|\theta-\theta^{*}\right\|_{2}$ & $0.2867 \pm 0.0721$ & $0.0897 \pm 0.0528$ & $0.0874 \pm 0.0525$ & $0.0761 \pm 0.0450$ & $0.0761 \pm 0.0451$ \\
\hline$n$ & $5.56 \pm 1.72$ & $7.09 \pm 1.38$ & $4.72 \pm 1.56$ & $7.44 \pm 0.94$ & N/A \\
\hline$t$ & $0.22 \pm 0.03$ & $1.35 \pm 0.05$ & $42.37 \pm 1.12$ & $3.42 \pm 0.08$ & N/A \\
\hline$T$ & $1.22 \pm 0.31$ & $9.56 \pm 1.81$ & $200.24 \pm 66.30$ & $25.41 \pm 3.19$ & N/A \\
\hline
\end{tabular}

Table 3: Correct decision boundary.

\begin{tabular}{|c|c|c|c|c|c|}
\hline & VT & VA1 & VA2 & EM & MLE \\
\hline$p$ & $0.747 \pm 0.031$ & $0.703 \pm 0.028$ & $0.702 \pm 0.028$ & $0.700 \pm 0.024$ & $0.699 \pm 0.024$ \\
\hline$\theta_{1}$ & $-2.4299 \pm 0.0753$ & $-2.4919 \pm 0.0596$ & $-2.4930 \pm 0.0594$ & $-2.4976 \pm 0.0531$ & $-2.4992 \pm 0.0532$ \\
\hline$\theta_{2}$ & $0.3944 \pm 0.1178$ & $0.0194 \pm 0.1099$ & $0.0173 \pm 0.1094$ & $0.0070 \pm 0.0944$ & $0.0039 \pm 0.0947$ \\
\hline$\left\|\theta-\theta^{*}\right\|_{1}$ & $0.4775 \pm 0.1653$ & $0.1382 \pm 0.0851$ & $0.1372 \pm 0.0846$ & $0.1179 \pm 0.0708$ & $0.1179 \pm 0.0710$ \\
\hline$\left\|\theta-\theta^{*}\right\|_{2}$ & $0.4058 \pm 0.1237$ & $0.1084 \pm 0.0657$ & $0.1076 \pm 0.0653$ & $0.0931 \pm 0.0558$ & $0.0931 \pm 0.0560$ \\
\hline$n$ & $14.16 \pm 3.60$ & $13.85 \pm 3.25$ & $12.23 \pm 2.86$ & $24.90 \pm 2.60$ & N/A \\
\hline$t$ & $0.72 \pm 0.15$ & $1.32 \pm 0.09$ & $39.01 \pm 1.26$ & $3.52 \pm 0.35$ & N/A \\
\hline$T$ & $10.13 \pm 3.01$ & $18.25 \pm 4.27$ & $478.19 \pm 117.67$ & $87.67 \pm 12.98$ & N/A \\
\hline
\end{tabular}

Table 4: Unknown weights. "Arbitrary" guess.

[17] D. Pollard. Strong consistency of k-means clustering. Ann. Statist., 9(1), 1981.

[18] L. Rabiner. A tutorial on Hidden Markov models and selected applications in speech recognition. Proc. IEEE, 77(2):257-286, 1989.

[19] L. Rabiner and B. Juang. Fundamentals of speech recognition. Prentice Hall, 1993.

[20] L. Rabiner, J. Wilpon, and B. Juang. A segmential k-means training procedure for connected word recognition. ATEST Tech. J., 64(3):21-40, 1986.

[21] M. Sabine and R. Gray. Global convergence and empirical consistency of the generalized Lloyd algorithm. IEEE Transaction on Information Theory, 32(2), 1986.

[22] V. Steinbiss, H. Ney, X. Aubert, S. Besling, C. Dugast, U Essen, D. Geller, R. Haeb-Umbach, R. Kneser, H. Meyer, M. Oerder, and B. Tran. The Philips research system for continuous-speech recognition. Philips Journal of Research, 49:317-352, 1995.

[23] N. Ström, L. Hetherington, T. Hazen, E. Sandness, and J. Glass. Acoustic modeling improvements in a segment-based speech recognizer. In Proc. IEEE ASRU Workshop, http://www.sls.lcs.mit.edu/sls/publications/1999/asru99-strom.pdf, 1999. 


\begin{tabular}{|c|c|c|c|c|c|}
\hline & VT & VA1 & VA2 & EM & MLE \\
\hline$p$ & $0.737 \pm 0.030$ & $0.699 \pm 0.026$ & $0.699 \pm 0.026$ & $0.699 \pm 0.023$ & $0.699 \pm 0.024$ \\
\hline$\theta_{1}$ & $-2.4526 \pm 0.0700$ & $-2.4987 \pm 0.0555$ & $-2.4987 \pm 0.0557$ & $-2.4991 \pm 0.0522$ & $-2.4992 \pm 0.0532$ \\
\hline$\theta_{2}$ & $0.3537 \pm 0.1114$ & $0.0058 \pm 0.1007$ & $0.0060 \pm 0.1021$ & $0.0038 \pm 0.0925$ & $0.0039 \pm 0.0947$ \\
\hline$\left\|\theta-\theta^{*}\right\|_{1}$ & $0.4212 \pm 0.1467$ & $0.1244 \pm 0.0782$ & $0.1263 \pm 0.0782$ & $0.1149 \pm 0.0701$ & $0.1179 \pm 0.0710$ \\
\hline$\left\|\theta-\theta^{*}\right\|_{2}$ & $0.3626 \pm 0.1146$ & $0.0978 \pm 0.0607$ & $0.0994 \pm 0.0607$ & $0.0907 \pm 0.0553$ & $0.0931 \pm 0.0560$ \\
\hline$n$ & $8.53 \pm 3.47$ & $6.01 \pm 2.44$ & $6.27 \pm 2.40$ & $11.89 \pm 4.24$ & N/A \\
\hline$t$ & $0.74 \pm 0.04$ & $1.36 \pm 0.05$ & $41.01 \pm 1.24$ & $3.53 \pm 0.06$ & N/A \\
\hline$T$ & $6.27 \pm 2.42$ & $8.13 \pm 3.19$ & $257.35 \pm 99.70$ & $41.84 \pm 14.81$ & N/A \\
\hline
\end{tabular}

Table 5: Unknown weights. Correct guess.

\begin{tabular}{|c|c|c|c|c|c|}
\hline & VT & VA1 & VA2 & EM & MLE \\
\hline$p$ & $0.737 \pm 0.029$ & $0.702 \pm 0.026$ & $0.700 \pm 0.026$ & $0.700 \pm 0.023$ & $0.699 \pm 0.024$ \\
\hline$\theta_{1}$ & $-2.4517 \pm 0.0689$ & $-2.4941 \pm 0.0573$ & $-2.4972 \pm 0.0556$ & $-2.4981 \pm 0.0526$ & $-2.4992 \pm 0.0532$ \\
\hline$\theta_{2}$ & $0.3549 \pm 0.1096$ & $0.0148 \pm 0.1050$ & $0.0087 \pm 0.1024$ & $0.0059 \pm 0.0930$ & $0.0039 \pm 0.0947$ \\
\hline$\left\|\theta-\theta^{*}\right\|_{1}$ & $0.4218 \pm 0.1459$ & $0.1327 \pm 0.0779$ & $0.1271 \pm 0.0780$ & $0.1164 \pm 0.0694$ & $0.1179 \pm 0.0710$ \\
\hline$\left\|\theta-\theta^{*}\right\|_{2}$ & $0.3637 \pm 0.1132$ & $0.1043 \pm 0.0606$ & $0.0999 \pm 0.0606$ & $0.0919 \pm 0.0548$ & $0.0931 \pm 0.0560$ \\
\hline$n$ & $8.16 \pm 3.40$ & $7.74 \pm 2.58$ & $6.54 \pm 2.22$ & $12.98 \pm 4.22$ & N/A \\
\hline$t$ & $0.74 \pm 0.04$ & $1.34 \pm 0.04$ & $41.12 \pm 1.74$ & $3.52 \pm 0.05$ & N/A \\
\hline$T$ & $5.97 \pm 2.36$ & $10.32 \pm 3.35$ & $268.96 \pm 91.44$ & $45.59 \pm 14.69$ & N/A \\
\hline
\end{tabular}

Table 6: Unknown weights. Correct boundary. 